\title{
Conhecimento Ecológico Local (CEL) na Avaliação do Estado de Conservação de Espécies de Interesse Socioeconômico: Integrando Saberes na Gestão do REVIS Ilha dos Lobos
}

\author{
Aline Kellermann ${ }^{1}$, Derien Vernetti Duarte ${ }^{2}$, Janina Huk ${ }^{3}$, Lais Gliesch Silva ${ }^{1}$, Roberta Aguiar dos Santos ${ }^{2}$, \\ Roberto Bruno Fabiano ${ }^{4}$ \& Walter Steenbock ${ }^{2}$
}

Recebido em 22/04/2020 - Aceito em 29/06/2020

1 Instituto Chico Mendes de Conservação da Biodiversidade/ICMBio, Refúgio de Vida Silvestre da Ilha dos Lobos/RS, Torres/RS, Brasil. CEP: 95.560-000. <aline.kellermann@icmbio.gov.br, laisgliesch@gmail.com>.

2 Instituto Chico Mendes de Conservação da Biodiversidade/ICMBio, Centro Nacional de Pesquisa e Conservação da Biodiversidade Marinha do Sudeste e Sul/CEPSUL, Itajaí/SC, Brasil. CEP: 88.301-445. <derienvernetti@yahoo.com.br, roberta.santos@icmbio.gov.br, walter.steenbock@icmbio.gov.br>.

3 Instituto Chico Mendes de Conservação da Biodiversidade/ICMBio, Centro Nacional de Pesquisa e Conservação da Sociobiodiversidade Associada a Povos e Comunidades Tradicionais/CNPT, Base Florianópolis/SC, Brasil. CEP: 88.053-700. <janina.huk@gmail.com>.

4 Consultor do Refúgio de Vida Silvestre da Ilha dos Lobos pelo Projeto de Áreas Marinhas e Costeiras Protegidas/GEF Mar. <rbfabiano@gmail.com>.

\begin{abstract}
RESUMO - A avaliação do estado de conservação de espécies da fauna brasileira, atribuição do Instituto Chico Mendes de Conservação da Biodiversidade (ICMBio), tem representado um esforço imenso e qualificado, agregando centenas de pesquisadores de diferentes instituições. Entretanto, neste processo, ainda é um desafio o envolvimento do conhecimento ecológico local (CEL) de usuários das espécies, o que tende a possibilitar tanto um arcabouço maior de informações para a avaliação quanto a ampliação da articulação social e institucional para a conservação. Essas possibilidades também são almejadas para a gestão participativa de unidades de conservação (UCs), visando maior eficiência e eficácia de suas metas em suas regiões de influência. Neste trabalho, relata-se a aplicação de uma metodologia que pode ser útil nestes dois escopos - no processo de avaliação do estado de conservação de espécies da fauna e na gestão de UCs. A partir da identificação, por pescadores artesanais, de 68 espécies aquáticas (entre teleósteos, elasmobrânquios e crustáceos) que ocorrem na região do Refúgio de Vida Silvestre (REVIS) Ilha dos Lobos/RS, foram selecionadas espécies consideradas importantes para a pesca nos ambientes de estuários/lagoas e oceânico (diferenciando-se os ambientes entre a UC e a costa, e em mar aberto). Sobre essas espécies, foram caracterizadas, de acordo com o CEL, a tendência populacional e as principais ameaças.
\end{abstract}

Palavras-chave: Conhecimento ecológico local; avaliação do estado de conservação de espécies; gestão participativa de unidades de conservação; risco de extinção.

\section{Local Ecological Knowledge (CEL) in Assessing the Conservation Status of Species of Socioeconomic Interest: Integrating Knowledge in the Management of REVIS Ilha dos Lobos}

ABSTRACT - The assessment of the conservation status of brazilian fauna, attributed by the Instituto Chico Mendes de Conservação da Biodiversidade (ICMBio), has been an immense and qualified effort, aggregating hundreds of researchers from different institutions. However, in this process, the involvement of the local ecological knowledge (LEK) of species that are caught by users still be a challenge, which tends to enable a greater information framework for the assessment and the expansion of the social and institutional articulation for conservation. These possibilities are also sought for the participative management of Protected Areas, aiming at greater efficiency and effectiveness of their goals in their regions. In this document, we report the application of a methodology that can be useful in these two scopes - in the assessment of the conservation status of the fauna process and in the management of protected areas. Based on the identification, by artisanal fishermen, of 68 aquatic species (among teleosts, elasmobranchs and crustaceans) that occur in the Wildlife Refuge llha 
dos Lobos (REVIS Ilha dos Lobos) region, species that were considered important for fishing were selected, considering estuaries/lagoons and ocean environments (differentiating the regions between the Refuge and the coast and in the open sea). On these species, according to the LEK, the population trend and the main threats were characterized.

Keywords: Local ecological knowledge; assessment of conservation status of species; participatory management of protected areas; extinction risk.

\title{
El Conocimiento Ecológico Local (CEL) en la Evaluación del Estado de Conservación de Especies de Interés Socioeconómico: Integración del Conocimiento en la Gestión de REVIS Ilha dos Lobos
}

\begin{abstract}
RESUMEN - La evaluación del estado de conservación de las especies de fauna brasileña, atribuida al Instituto Chico Mendes para la Conservación de la Biodiversidad (ICMBio), ha representado un esfuerzo inmenso y calificado, reuniendo a cientos de investigadores de diferentes instituciones. Sin embargo, en este proceso, la inclusión del conocimiento ecológico local (CEL) de los usuarios de las especies continua siendo un desafío, que posibilita tanto un marco más amplio de información para la evaluación como la expansión de la articulación social e institucional para la conservación. Estas posibilidades también están orientadas al manejo participativo de las Unidades de Conservación (UC), buscando una mayor eficiencia y efectividad de sus objetivos en sus regiones de influencia. En este trabajo informamos la aplicación de una metodología pertinente en estos dos ámbitos - en el proceso de evaluación del estado de conservación de las especies de fauna y en el manejo de las UC. A partir de la identificación, por parte de pescadores artesanales, de 68 especies acuáticas (entre teleósteos, elasmobranquios y crustáceos) que se dan en el Refugio de Vida Silvestre (REVIS) Ilha dos Lobos/RS, se seleccionaron especies como importantes para la pesca en los ambientes de esteros/ lagunas y océano (diferenciando los ambientes entre la UC y la costa, y en mar abierto). Sobre estas especies, según el CEL, se caracterizó la tendencia poblacional y las principales amenazas.
\end{abstract}

Palabras clave: Conocimiento ecológico local; evaluación del estado de conservación de las especies; manejo participativo de unidades de conservación; riesgo de extinción.

\section{Introdução}

O processo de avaliação do estado de conservação das espécies é a base para a definição da lista de espécies ameaçadas de extinção no Brasil, bem como do nível e natureza das ameaças. Com essa avaliação, é possível elaborar políticas públicas que visem à redução das ameaças e à conservação das espécies e seus habitat (Brasil 2014a).

No Brasil, cabe ao Ministério do Meio Ambiente a avaliação do estado de conservação das espécies, a partir de duas autarquias federais: o Jardim Botânico do Rio de Janeiro (JBRJ), para as espécies da flora brasileira; e o Instituto Chico Mendes de Conservação da Biodiversidade (ICMBio) (ICMBio 2013, Brasil 2014a), para todas as espécies de vertebrados que ocorrem em território nacional e para alguns grupos de invertebrados selecionados.

A metodologia do processo de avaliação das espécies da fauna segue a orientação do protocolo desenvolvido pela União Internacional para Conservação da Natureza (UICN), aplicado em diversos países (IUCN 2019) conforme diretrizes da Instrução Normativa do ICMBio $n^{\circ} 34$ de 2013. Esse processo é executado em etapas, iniciando-se com a compilação $e$ organização de informações sobre cada espécie por meio do Sistema de Avaliação do Estado de Conservação da Biodiversidade do ICMBio (SALVE), passando por consulta ampla (pública) $e$ direta aos especialistas da comunidade científica para revisão das informações e, após, pela oficina de avaliação do risco de extinção das espécies, com a presença de especialistas de diversas instituições. Então, é feita a validação dos resultados da avaliação, quando se checa a aplicação do método. Finalmente, promove-se a publicação e divulgação dos resultados (Souza et al. 2018).

O atual processo de avaliação do risco de extinção da fauna brasileira, coordenado pelo ICMBio, teve seu primeiro ciclo iniciado em 
2009 e finalizado em 2014. Foram avaliadas 12.254 espécies, em 73 oficinas de trabalho com a participação de mais de 1.270 especialistas da comunidade científica brasileira e estrangeira, provenientes de mais de 250 instituições (ICMBio 2018).

O segundo ciclo do processo de avaliação foi iniciado em 2015, agregando a avaliação de novos grupos de invertebrados e de vertebrados recémdescritos, e a reavaliação de todas as espécies avaliadas no ciclo anterior, com o acréscimo das informações produzidas nos últimos anos (Souza et al. 2018). Em 2018, cerca de 5 mil espécies já tinham sido reavaliadas, havendo a indicação de que poderão ser avaliadas mais de 13 mil espécies neste novo ciclo (Souza et al. 2018).

Segundo esses autores, dentre os táxons que já foram reavaliados e cujos resultados foram validados, o motivo mais frequente para a mudança de categoria de ameaça foi a disponibilidade de novas ou melhores informações, ou seja, o aumento do conhecimento.

Assim, para que a avaliação seja cada vez mais precisa, é necessário buscar estratégias para sanar ao menos duas lacunas. Uma delas consiste basicamente na qualidade e quantidade de publicações sobre as espécies, material básico da avaliação: quanto mais qualificados e em maior número os trabalhos de pesquisa sobre as espécies, mais acurada será a avaliação, o que indica a necessidade de apoio e disponibilização de recursos a esse tipo de investigação. A segunda lacuna está relacionada ao envolvimento do conhecimento ecológico local (CEL) no processo de avaliação. Usuários de espécies e habitat como os pescadores em relação às espécies-alvo de captura - tendem a possuir elevado e complexo conhecimento sobre a ecologia, os ciclos de vida, as ameaças e as tendências populacionais dessas espécies, de forma temporal e espacialmente contextualizada (Diegues 2000, Hanazaki et al. 2013, Begossi et al. 2017). Entretanto, ainda são insuficientes os estudos em etnoecologia que caracterizam esses conhecimentos em relação ao conjunto de espécies e suas regiões de ocorrência. Paralelamente, ainda não há uma metodologia clara e institucionalizada, no Brasil, para o envolvimento direto de usuários de espécies no processo de avaliação.

Buscar esse envolvimento é uma das diretrizes da Convenção da Diversidade Biológica
(CDB), formulada em território brasileiro durante a Eco ou Rio-92 e ratificada pelo país nos anos seguintes (Brasil 1998). Com base nessa preocupação, foi desenvolvida pelo ICMBio uma proposta metodológica de agregação do CEL sobre a ictiofauna no âmbito das unidades de conservação federais, em oficina conduzida pela Coordenação Geral de Pesquisa e Monitoramento da Biodiversidade (CGPEq) e pela Coordenação Geral de Estratégias para a Conservação (CGCON), em abril de 2018. Tal metodologia foi aplicada de forma ligeiramente adaptada, como experiência piloto, na oficina descrita neste trabalho, no contexto da gestão do Refúgio de Vida Silvestre (REVIS) Ilha dos Lobos, na cidade de Torres/RS.

Afinal, agregar usuários de espécies e habitat e seus conhecimentos não é fundamental apenas para o processo de avaliação do estado de conservação de espécies, mas também no âmbito da gestão das UCs, uma vez que é de grande importância buscar a participação desses usuários em seus conselhos e na elaboração de seus instrumentos de gestão, conforme determina o Sistema Nacional de Unidades de Conservação (SNUC) (Brasil 2000) e o Decreto $n^{\circ} 4340 / 2002$ (Brasil 2002), que o regulamenta. Essa participação, longe de se constituir uma concessão, visa justamente o maior envolvimento social $e$, consequentemente, a maior possibilidade de conservação efetiva da natureza.

O REVIS Ilha dos Lobos é a única ilha oceânica do litoral do estado do Rio Grande do Sul. Criado como unidade de conservação em 1983, então como Reserva Ecológica, teve seu Conselho formado apenas em 2016. Nesse processo de formação, deu-se especial importância à articulação entre diferentes setores da sociedade, bem como à abrangência regional de sua atuação, com reflexo ou impacto no REVIS e nas populações de pinípedes (lobos e leões marinhos) que nele habitam (Kellermann 2018).

Hoje, o Conselho tem atuado efetivamente na gestão da unidade, a partir de um plano de ação construído coletivamente. Essa atuação é a base para a elaboração participativa do plano de manejo do REVIS, em fase inicial de construção (Kellermann 2018). Nesse processo, a representação do setor pesqueiro se dá pelas Colônias de Pescadores de Torres Z-7/RS e de Passo de Torres Z-18/SC, e tem sido muito importante para a gestão local e regional da unidade. 
Considerando o contexto exposto, em 17 de janeiro de 2019 foi realizada, na Colônia de Pesca Z-7, uma oficina de diagnóstico do conhecimento dos pescadores da região do REVIS Ilha dos Lobos sobre a ictiofauna e sua relação com as ações antrópicas. O objetivo da oficina foi gerar informações que servissem de subsídios para a elaboração de instrumentos de gestão adequados no âmbito da UC e que contribuíssem para novas políticas públicas, em especial em relação à avaliação do estado de conservação de espécies de peixes. A metodologia aplicada na oficina utilizou ferramentas da etnoecologia, envolvendo, em especial, elementos da pesquisa qualitativa (Minayo 1994), o mapeamento mental (Geilfus 2002, Steenbock \& Medeiros 2017) e o diagnóstico rápido participativo (Brose 2001).

\section{Material e Métodos}

Em etapa preliminar, e com base em dados secundários, foi elaborada uma lista de espécies de peixes majoritariamente marinhos (Actinopterygii $\mathrm{n}=50$, peixes ósseos; e Elasmobranchii $\mathrm{n}=16$, peixes cartilaginosos) com potencial de ocorrência na região do REVIS Ilha dos Lobos. Na elaboração dessa lista, não houve a preocupação de garantir que ela fosse completa, mas, sim, que viesse a servir de base para o diagnóstico participativo.

Foram impressas fichas contendo o nome popular, o nome científico e a foto de cada espécie da lista, para uso na oficina. Foi também desenhado um mapa básico da região do REVIS, em papel pardo de aproximadamente 2,5m x 2,5m. $\mathrm{Na}$ elaboração desse mapa, foram indicados a linha de costa, o território do REVIS, o morro do Farol, o rio Mampituba (principal rio da região, que divide os estados de Santa Catarina e Rio Grande do Sul), o Parque Estadual de Itapeva e algumas lagoas, sem preocupação com a precisão cartográfica nem com a indicação de demais elementos.

Mobilizada no âmbito da gestão do REVIS, $e$ tendo o objetivo claramente definido nos convites direcionados aos pescadores artesanais das Colônias de Pescadores de Torres Z-7/RS e de Passo de Torres Z-18/SC, a oficina foi iniciada às 14 horas do dia 17 de janeiro de 2019 , com previsão de término às 17 horas. A equipe de condução da oficina foi composta por seis pessoas, com atribuições discutidas e acordadas em reunião prévia (coordenação, moderação, relatoria, relatoria visual e apoio à discussão com os pescadores). Participaram da oficina 11 pescadores. $\mathrm{O}$ registro da presença dos pescadores foi feito em lista com nome, contato, idade e o tempo que cada participante pesca na região.

Após a apresentação dos objetivos da oficina e sua contextualização na gestão do REVIS, o mapa previamente elaborado foi disposto no chão, no centro da sala. Foi proposto, então, que todos os presentes identificassem os elementos do mapa, dessem nomes aos elementos e o completassem com demais referências, utilizando canetas de marcação (pinceis atômicos). O objetivo dessa atividade foi promover a identificação cartográfica de cada pescador com o ambiente sob análise, por meio de mapeamento mental (Geilfus 2002, Steenbock \& Medeiros 2017). Em seguida, foi utilizada a ferramenta de checklist (Brose 2001, Steenbock \& Medeiros 2017), contextualizada ao mapeamento mental. As fichas das espécies foram oferecidas ao grupo, com a proposta de identificação visual das espécies, da ratificação ou adequação dos nomes populares em relação a cada foto, e da disposição das espécies no mapa, de acordo com sua região de ocorrência principal (Figura 1A). Foi proposto também que, caso houvesse fichas com espécies não ocorrentes na região do mapa, que estas fossem separadas e retiradas e, caso houvesse espécies importantes para os pescadores que não haviam sido indicadas, que estas fossem identificadas em fichas específicas. Durante a atividade, foram discutidos vários aspectos em relação à ocorrência das espécies e sua relação com a área do REVIS.

Então, foi disposto um cesto no centro do mapa, propondo-se aos pescadores que depositassem em seu interior as espécies consideradas importantes (Figura 1B). A pergunta utilizada foi "que espécies são importantes para você?", sem detalhamento de importância para comercialização, para consumo ou qualquer outra condição. Também não foi detalhada a importância diferencial dada pelo pescador individualmente, ou seja, foram colocadas no cesto as fichas de todas as espécies consideradas importantes pelo conjunto dos pescadores presentes na oficina.

As fichas não colocadas no cesto foram retiradas do mapa, recolocando-se então sobre ele apenas as fichas que haviam sido incluídas no cesto. A premissa para essa retirada foi de que a análise da tendência populacional, da importância socioeconômica e das ameaças (atividades 
realizadas em seguida) seria mais informativa para espécies consideradas importantes. As fichas dessas espécies foram agrupadas em três regiões: espécies de alto mar, espécies que ocorrem mais próximo à costa e espécies das lagoas.

Foram distribuídos então círculos adesivos verdes, amarelos e vermelhos a cada pescador, propondo-se uma avaliação da tendência populacional, da seguinte forma: para cada espécie e considerando o período dos últimos cinco anos (2014-2019), caso o pescador considerasse, pela sua própria percepção, que sua quantidade (população) tinha aumentado, deveria colar na ficha um adesivo verde; se a população vinha se mantendo estável, seria utilizado um adesivo amarelo; e, caso a população viesse diminuindo, um adesivo vermelho. É importante notar que o período de tempo proposto para a avaliação - de cinco anos - não foi baseado no tempo geracional das espécies selecionadas, mas considerando apenas uma referência temporal recente. Levou-se em conta que o método não seria robusto o suficiente para a avaliação precisa associada a diferentes ( $e$ maiores) períodos de tempo. Para tanto, a aplicação de metodologias participativas de diagnóstico como a "linha do tempo" e "histórias orais" (Geilfus 2002, Steenbock \& Medeiros 2017) e a realização complementar de entrevistas individuais poderia ser útil, o que demandaria, porém, um maior período de tempo para a oficina.

Ao final dessa atividade, cada espécie considerada importante teve sua tendência populacional caracterizada, considerando a perspectiva individual de cada pescador (Figura 1C). Como havia pescadores que atuavam somente nas lagoas e não no mar, e vice-versa, foi proposto que cada pescador só avaliasse a tendência populacional das espécies que costuma pescar. Foi também proposto que, caso o pescador não se sentisse apto a avaliar a tendência populacional de alguma espécie, que se eximisse da avaliação. Durante esse processo, vários aspectos relacionados à variação populacional das espécies em tela, nos últimos cinco anos, foram discutidos e registrados.

Para a caracterização do grau de importância socioeconômica das espécies selecionadas, foi realizadoum ranqueamento (ranking), considerando separadamente os três agrupamentos de espécies (mar, costa e lagoas). No ranqueamento, em cada agrupamento foram distribuídos 10 grãos de milho para cada pescador e proposto que cada um dispusesse a quantidade de grãos correspondente ao grau de importância que dava a cada espécie junto à sua ficha (Figura 1D). Novamente foi proposto que só participasse do ranqueamento, em cada agrupamento, os pescadores que costumavam pescar as espécies daquele agrupamento.

Foi então proposto que se identificassem as principais ameaças às espécies de cada agrupamento. Originalmente, essa atividade seria realizada considerando as ameaças às espécies ranqueadas como mais importantes. Entretanto, durante as discussões das atividades anteriores, ficou explícito que as ameaças estavam relacionadas aos agrupamentos de espécies, conforme sua região de ocorrência principal. Foi solicitado a cada pescador que identificasse as principais ameaças a cada agrupamento de espécies, em rodadas específicas por agrupamento. As ameaças citadas foram descritas em fichas e dispostas junto aos agrupamentos (Figuras 1E e 1F). Assim como ocorreu nas etapas anteriores, durante a definição das ameaças foram estimuladas e registradas as discussões, incentivando-se o debate sobre as origens das ameaças e sobre as perspectivas de gestão para reduzi-las.

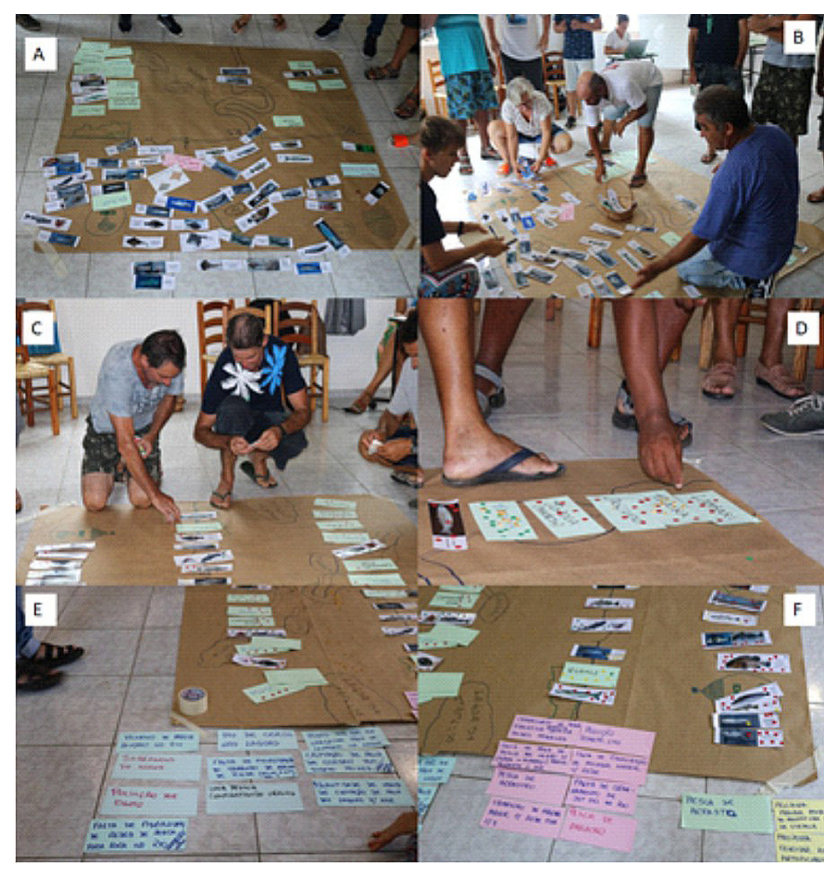

Figura 1 - Atividade de zoneamento das espécies (A); Identificação de espécies consideradas importantes (B); Análise da tendência populacional de espécies consideradas importantes (C); Ranqueamento de espécies em função do grau de importância socioeconômica (D); Identificação das principais ameaças a espécies da ictiofauna, de acordo com pescadores artesanais da região (E e F). 


\section{Resultados e Discussão}

\section{Caracterização do grupo de pescadores}

Os 11 pescadores que participaram da oficina definiram-se como pescadores artesanais que atuam especialmente próximo à costa $e$ às lagoas e estuários, sendo que alguns, eventualmente, trabalham embarcados na pesca industrial. A idade média do grupo é de 55 anos (de 47 a 64 anos) e o tempo médio de pesca na região é de 33 anos (de 15 a 50 anos). Do grupo, nove pescadores eram do sexo masculino e dois do sexo feminino.

\section{Mapa mental da região do REVIS Ilha dos Lobos}

A proposição da elaboração do mapa mental como atividade inicial serviu para estimular a participação e a construção coletiva de todos os pescadores presentes. A identificação dos pescadores com o mapa se deu rapidamente, sendo marcante para isso a reorientação de posição do mapa no chão, de acordo com a posição relativa da sala da oficina ao ambiente marinho: quando isso foi proposto por um dos pescadores, todos se manifestaram identificando as referências no mapa. Foram incluídos no mapa, pelos pescadores, nomes de lagoas, canais (entre as lagoas, entre as lagoas e o mar, e entre as lagoas e o rio Mampituba), canais que foram assoreados (e não existem mais) e a ocorrência de um parcel (de aproximadamente $4 \mathrm{~km}$, na mesma latitude de Torres), entre outras referências cartográficas.

\section{Ocorrência de espécies da ictiofauna reconhecidas na região do REVIS Ilha dos Lobos}

A partir da lista prévia de espécies e das inserções de novas espécies, e considerando a área mapeada, foram identificadas, pelos pescadores presentes na oficina, 68 espécies aquáticas (entre teleósteos, elasmobrânquios e crustáceos) (Tabela 1).

Tabela 1 - Ocorrência de espécies da ictiofauna na região do REVIS Ilha dos Lobos, de acordo com pescadores artesanais.

\begin{tabular}{|c|c|c|c|c|}
\hline Família/Espécie & Nome comum & $\mathbf{O}^{*}$ & $\mathbf{C}^{*}$ & $\mathbf{E}^{*}$ \\
\hline \multicolumn{5}{|l|}{ Actinopterygii } \\
\hline \multicolumn{5}{|l|}{ Ordem Atheriniformes } \\
\hline \multicolumn{5}{|l|}{ Família Atherinopsidae } \\
\hline Odontesthes argentinensis (Valenciennes 1835) & peixe-rei & & $\mathrm{x}$ & \\
\hline Atherinella brasiliensis (Quoy \& Gaimard 1825) & peixe-rei & & & $\mathrm{x}$ \\
\hline \multicolumn{5}{|l|}{ Ordem Carangiformes } \\
\hline \multicolumn{5}{|l|}{ Família Carangidae } \\
\hline Caranx hippos (Linnaeus 1766) & xaréu & 0 & & \\
\hline Caranx latus Agassiz 1831 & xerelete & 0 & & \\
\hline \multicolumn{5}{|l|}{ Família Stromateidae } \\
\hline Preprilus paru (Linnaeus 1758) & gordinho & 0 & & \\
\hline Stromateus brasiliensis Fowler 1906 & pampo, solteiro & $\mathrm{x}$ & & \\
\hline \multicolumn{5}{|l|}{ Ordem Characiformes } \\
\hline \multicolumn{5}{|l|}{ Família Characidae } \\
\hline Astyanax sp. & lambari** & & & $\mathrm{x}$ \\
\hline Cyphocharax sp. & birú $* *$ & & & $\mathrm{x}$ \\
\hline \multicolumn{5}{|l|}{ Família Erythrinidae } \\
\hline Hoplias sp. & traíra** & & & $\mathrm{x}$ \\
\hline
\end{tabular}




\begin{tabular}{|c|c|c|c|c|}
\hline \multicolumn{5}{|l|}{ Ordem Clupeiformes } \\
\hline \multicolumn{5}{|l|}{ Família Clupeidae } \\
\hline Sardinella brasiliensis Valenciennes 1847 & sardinha-verdadeira & $\mathrm{X}$ & & \\
\hline Brevoortia pectinata (Jenyns 1842) & savelha, manjubão & & $\mathrm{x}$ & \\
\hline Harengula cluopeola (Cuvier 1829) & sardinha-cascuda & 0 & & \\
\hline Platanichthys platana (Regan 1917) & sardinha-manjuba & 0 & & \\
\hline Ramnogaster arcuata (Jenyns 1842) & sardinha & 0 & & 0 \\
\hline \multicolumn{5}{|l|}{ Família Engraulidae } \\
\hline Engraulis anchoita Hubbs \& Marini 1935 & anchoita & $\mathrm{X}$ & & \\
\hline Anchoa tricolor (Spix \& Agassiz 1829) & dente-cão & & & 0 \\
\hline Anchoa marinii Hildebrand 1943 & manjuba & & & 0 \\
\hline Lycengraulis grossidens (Spix \& Agassiz 1829) & manjubão & $\mathrm{X}$ & & \\
\hline \multicolumn{5}{|l|}{ Ordem Gadiformes } \\
\hline \multicolumn{5}{|l|}{ Família Phycidae } \\
\hline Urophycis brasiliensis (Kaup 1858) & brota & $\mathrm{X}$ & & \\
\hline \multicolumn{5}{|l|}{ Ordem Perciformes } \\
\hline \multicolumn{5}{|l|}{ Família Centropomidae } \\
\hline Centropomus parallelus Poey 1860 & robalo-peva & & $\mathrm{X}$ & \\
\hline Centropomus undecimalis (Bloch 1792) & robalo** & & $\mathrm{X}$ & $\mathrm{X}$ \\
\hline \multicolumn{5}{|l|}{ Família Cichlidae } \\
\hline Crenicichla lepidota Heckel 1840 & joana, badejo** & & & $\mathrm{X}$ \\
\hline Geophagus sp. & cará ** & & & $\mathrm{X}$ \\
\hline \multicolumn{5}{|l|}{ Família Ephippidae } \\
\hline Chaetodipterus faber (Broussonet 1782) & enxada, paru & 0 & & \\
\hline \multicolumn{5}{|l|}{ Família Epinephelidae } \\
\hline Epinephelus marginatus (Lowe 1834) & garoupa & $\mathrm{X}$ & & \\
\hline \multicolumn{5}{|l|}{ Família Haemulidae } \\
\hline Orthopristis rubra (Cuvier 1830) & cocoroca & $\mathrm{X}$ & & \\
\hline \multicolumn{5}{|l|}{ Família Mugilidae } \\
\hline Mugil liza Valenciennes 1836 & tainha & & $\mathrm{X}$ & $\mathrm{X}$ \\
\hline Mugil curema Valenciennes 1836 & parati & 0 & & \\
\hline Mugil gaimardianus Desmarest 1831 & tainhota & 0 & & \\
\hline \multicolumn{5}{|l|}{ Família Pomatomidae } \\
\hline Pomatomus saltatrix (Linnaeus 1766) & anchova, enchova & $\mathrm{X}$ & & \\
\hline \multicolumn{5}{|l|}{ Família Priacanthidae } \\
\hline Priacanthus arenatus Cuvier 1829 & olho-de-boi, olho-de-cão & 0 & & \\
\hline \multicolumn{5}{|l|}{ Família Scianidae } \\
\hline Cynoscion guatucupa (Cuvier 1830) & pescada-olhuda, maria-mole & $\mathrm{X}$ & & \\
\hline Cynoscion jamaicensis (Vaillant \& Bocourt 1883) & goete & 0 & & \\
\hline Macrodon atricauda (Günther 1880) & pescadinha & $\mathrm{X}$ & & \\
\hline Menticirrhus litorallis (Holbrook 1847) & imbitarra-branca & $\mathrm{X}$ & & \\
\hline
\end{tabular}




\begin{tabular}{|c|c|c|c|c|}
\hline Menticirrhus americanus (Linnaeus 1758) & imbitarra & $\mathrm{X}$ & & \\
\hline Micropogonias furnieri (Desmarest 1823) & corvina, cascote & & $\mathrm{X}$ & \\
\hline Paralonchurus brasiliensis (Steindachner 1875) & maria-luiza & $\mathrm{X}$ & & \\
\hline Pogonias cromis (Linnaeus 1766) & miragaia, burriquete (quando jovem) & $\mathrm{X}$ & & \\
\hline Umbrina canosai Berg 1895 & castanha & $\mathrm{X}$ & & \\
\hline \multicolumn{5}{|l|}{ Família Sparidae } \\
\hline Pagrus pagrus (Linnaeus 1758) & pargo-rosa & 0 & & \\
\hline \multicolumn{5}{|l|}{ Família Trichiuridae } \\
\hline Trichiurus lepturus Linnaeus 1758 & peixe-espada & 0 & & \\
\hline \multicolumn{5}{|l|}{ Ordem Pleuronectiformes } \\
\hline \multicolumn{5}{|l|}{ Família Paralichtyidae } \\
\hline Paralichthys orbignyanus (Valenciennes 1839) & linguado-vermelho & & & $\mathrm{X}$ \\
\hline Paralichthys patagonicus Jordan 1889 & linguado-branco & & & $\mathrm{X}$ \\
\hline \multicolumn{5}{|l|}{ Família Pleuronectidae } \\
\hline Oncopterus darwini Steindachner 1874 & linguado & 0 & & \\
\hline \multicolumn{5}{|l|}{ Ordem Scombriformes } \\
\hline \multicolumn{5}{|l|}{ Família Scombridae } \\
\hline Katsuwonus pelamis (Linnaeus 1758) & bonito-listrado & 0 & & \\
\hline \multicolumn{5}{|l|}{ Ordem Siluriformes } \\
\hline \multicolumn{5}{|l|}{ Família Ariidae } \\
\hline Genidens barbus (Lacepède 1803) & bagre-branco & & $\mathrm{X}$ & \\
\hline Genidens genidens (Cuvier 1829) & guri & & & $\mathrm{x}$ \\
\hline Genidens machadoi (Miranda Ribeiro 1918) & bagre & & & 0 \\
\hline \multicolumn{5}{|l|}{ Família Heptapteridae } \\
\hline Rhamdia sp. & jundiá*** & & & $\mathrm{x}$ \\
\hline \multicolumn{5}{|l|}{ Família Loricariidae } \\
\hline Hypostomus sp. & cascudo** & & & $\mathrm{x}$ \\
\hline Loricariichthys anus (Valenciennes 1835) & violinha-da-lagoa** & & & $\mathrm{x}$ \\
\hline \multicolumn{5}{|l|}{ Ordem Synabranchiformes } \\
\hline \multicolumn{5}{|l|}{ Família Synbranchidae } \\
\hline Synbranchus marmoratus Bloch 1795 & mussum** & & & $\mathrm{X}$ \\
\hline \multicolumn{5}{|l|}{ Ordem Syngnathiformes } \\
\hline \multicolumn{5}{|l|}{ Família Syngnathidae } \\
\hline Espécie não identificada & peixe-rei-agulha & 0 & & \\
\hline \multicolumn{5}{|l|}{ Elasmobranchii } \\
\hline \multicolumn{5}{|l|}{ Ordem Carcharhiniformes } \\
\hline \multicolumn{5}{|l|}{ Família Sphyrnidae } \\
\hline Sphyrna lewini (Griffith \& Smith 1834) & tubarão-martelo & $\mathrm{X}$ & & \\
\hline \multicolumn{5}{|l|}{ Ordem Lamniformes } \\
\hline \multicolumn{5}{|l|}{ Família Odontaspididae } \\
\hline Carcharias taurus Rafinesque 1810 & mangona & 0 & & \\
\hline
\end{tabular}




\begin{tabular}{|c|c|c|c|}
\hline \multicolumn{4}{|l|}{ Ordem Myliobatiformes } \\
\hline \multicolumn{4}{|l|}{ Família Myliobatidae } \\
\hline Myliobatis freminvilei Lesueur 1824 & raia-sapo, ticonha & 0 & \\
\hline \multicolumn{4}{|l|}{ Ordem Rajiformes } \\
\hline \multicolumn{4}{|l|}{ Família Arhynchobatidae } \\
\hline Atlantoraja castelnaui (Miranda Ribeiro 1907) & raia-chita $* *$ & 0 & \\
\hline \multicolumn{4}{|l|}{ Família Dasyatidae } \\
\hline Espécie não identificada & raia-manteiga ** & 0 & \\
\hline \multicolumn{4}{|l|}{ Família Gymnuridae } \\
\hline Gymnura altavela (Linnaeus 1758) & raia-borboleta & 0 & \\
\hline \multicolumn{4}{|l|}{ Ordem Rhinopristiformes } \\
\hline \multicolumn{4}{|l|}{ Família Rhinobatidae } \\
\hline Pseudobatos horkelli (Müller \& Henle 1841) & viola & $\mathrm{X}$ & \\
\hline \multicolumn{4}{|l|}{ Família Trygonorrhinidae } \\
\hline Zapteryx brevirostris (Müller \& Henle 1841) & banjo & 0 & \\
\hline \multicolumn{4}{|l|}{ Ordem Squatiniformes } \\
\hline \multicolumn{4}{|l|}{ Família Squatinidae } \\
\hline Squatina guggenheim Marini 1936 & cação-anjo, cação-anjo-espinhudo & 0 & \\
\hline Squatina occulta Vooren \& da Silva 1991 & cação-anjo & $\mathrm{X}$ & \\
\hline \multicolumn{4}{|l|}{ Crustacea } \\
\hline \multicolumn{4}{|l|}{ Ordem Decapoda } \\
\hline \multicolumn{4}{|l|}{ Família Penaeidae } \\
\hline Xiphopenaeus kroyeri Heller 1862 & camarão-sete-barbas $* *$ & 0 & \\
\hline Penaeus sp. & camarão-pistola** $^{* *}$ & & $\mathrm{X}$ \\
\hline Penaeus paulensis (Perez-Farfante 1967) & camarão-rosa** & & $\mathrm{X}$ \\
\hline \multicolumn{4}{|l|}{ Família Portunidae } \\
\hline Callinectes sp. & siri** & & $\mathrm{x}$ \\
\hline
\end{tabular}

* $\mathrm{O}=$ espécies com predominância de ocorrência no oceano, em mar aberto; $\mathrm{C}=$ espécies com predominância de ocorrência no oceano, entre o REVIS e a costa; $\mathrm{E}=$ espécies com predominância de ocorrência nos estuários/lagoas;

$\mathrm{X}=$ espécies selecionadas como importantes;

$0=$ espécies que ocorrem nos ambientes, mas não selecionadas como importantes.

**Espécies inseridas na oficina sem identificação de nomenclatura científica, sendo realizado posteriormente, por meio de análise bibliográfica de ocorrência em regióes próximas e/ou na região Sul.

Obs.: para as espécies inseridas durante a oficina (neste caso, $24 \%$ do total, sendo a maior parte de peixes continentais e crustáceos, não relacionados na lista prévia, que continha apenas espécies de peixes marinhos), seria ideal a identificação com nomenclatura científica durante a própria atividade, utilizando imagens online a partir de repositórios de imagens com identificação confiável, para exposição e discussão com os participantes. Para tanto, seria necessário prever um período de tempo maior para a oficina.

\section{Importância socioeconômica e tendência populacional das espécies}

Conforme descrito no item Material e Métodos, as fichas relacionadas às espécies consideradas importantes para os pescadores presentes na oficina foram colocadas dentro de um cesto, filtrando-as, assim, do conjunto total de espécies identificadas.

Essas espécies estão indicadas na Tabela 2, por região de predominância de ocorrência caracteri- 
zada no mapa. Cada região apresenta o ranking de importância das espécies (número total de grãos de milho utilizados para votação em cada espécie), a importância relativa (ranking de importância expresso em percentagem) e a tendência populacional das espécies consideradas importantes (expressa em percentagem de pescadores que avaliaram se as populações estão diminuindo, estáveis ou aumentando, nos últimos cinco anos).

Na Figura 2 apresentam-se os dados da Tabela 2, sistematizados em gráficos. Das 18 espécies consideradas importantes socioeconomicamente e com predominância de ocorrência nas lagoas/estuários, a maioria (15) foi considerada, por unanimidade, em declínio populacional nos últimos cinco anos. Nesse ambiente, a tainha (Mugil liza) foi considerada com tendência de aumento populacional por $100 \%$ dos pescadores que fizeram a avaliação. O guri (Genidens genidens) também foi considerado em tendência de aumento populacional pela maioria dos pescadores que participaram da avaliação. Já o linguado-vermelho (Paralichtys orbignyanus) foi considerado com estoque estável ou em processo de redução (Figura 2A).

Para as espécies com predominância de ocorrência na região costeira entre o REVIS e o continente (e que também podem adentrar os estuários), o número total de indicações em relação à tendência do estoque envolve um número maior de indicações de aumento e estabilidade (Figura 2B). Chama atenção o fato de a tainha (Mugil liza), nesse ambiente,

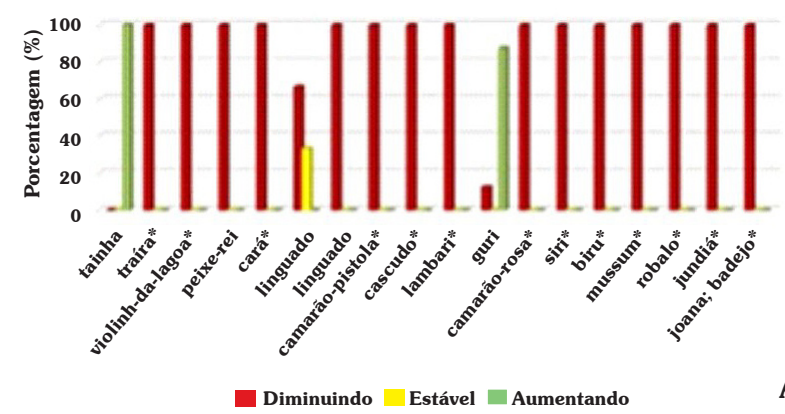

A

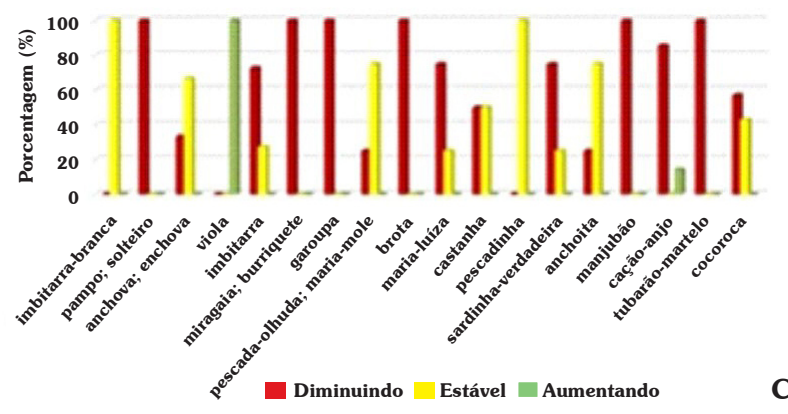

ser considerada também em situação estável ou em redução de estoque, se comparado à unânime consideração da tainha como com estoques aumentando na região estuarina. Outra unanimidade de considerações foi em relação aos estoques do peixe-rei (Odontesthes argentinensis), do bagre-branco (Genidens barbus) e da savelha (Brevoortia pectinata), respectivamente, como diminuindo, aumentando e estável.

Em relação às espécies consideradas predominantemente oceânicas, a maior parte das indicações considerou os estoques entre estável e em diminuição, nos últimos cinco anos (Figura 2C). Apenas o cação-anjo (Squatina occulta) e a viola (Pseudobatos horkelli) - ambos elasmobrânquios classificados oficialmente como ameaçados de extinção (Portaria MMA n ${ }^{\circ} 445 / 2014$ ) - chegaram a ter indicações de estoque aumentando. No caso da viola, $100 \%$ dos pescadores que a avaliaram nesse ambiente consideram que seu estoque está aumentando (Figura 2C).

Durante a análise da tendência populacional, vários comentários foram registrados, indicando diferentes percepções e interpretações sobre as espécies e as políticas públicas relacionadas ao ordenamento pesqueiro. Esses comentários - assim como outros colocados durante essa atividade não chegaram a ser discutidos, devido à limitação de tempo da oficina. Entretanto, seja para um maior entendimento do ordenamento pesqueiro, seja para a aproximação dos pescadores ao processo de avaliação do estado de conservação das espécies e à gestão do REVIS Ilha dos Lobos, considera-se essa discussão bastante importante.

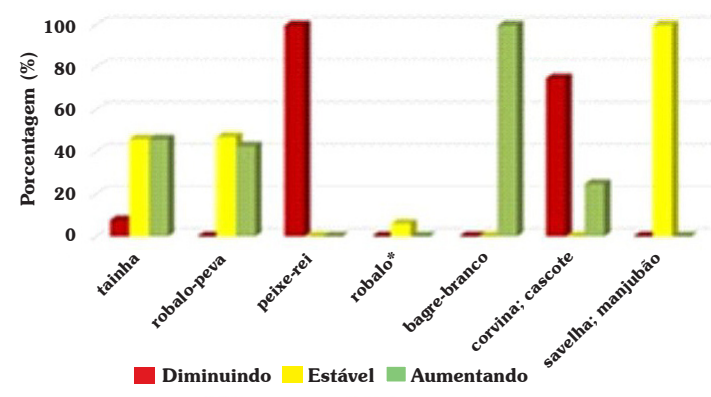

Figura 2 - Distribuição de tendência populacional para cada espécie, de acordo com os pescadores artesanais da região do REVIS, entre as categorias pescadas em lagoas $e$ estuários (A); entre as categorias pescadas na região costeira, entre o REVIS e o continente, podendo entrar nos estuários (B); e entre as categorias pescadas em mar aberto $(\mathrm{C})$. 


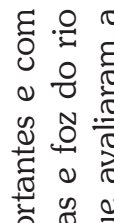

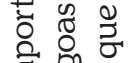

घ.

$\frac{0}{\pi} \frac{\pi}{8} \frac{0}{0}$

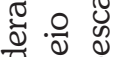

这

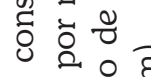

\%ै.

,

कु

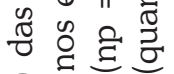

운 중 원

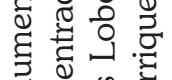

के

$\leftarrow \cdot \sigma \pi$

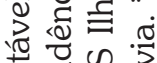

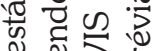

ข

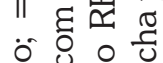

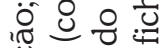

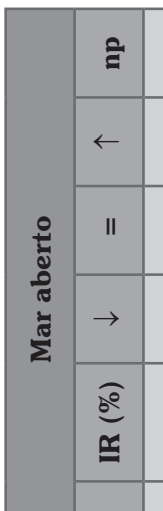

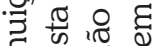

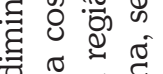

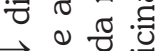

$\geq \infty$ 电

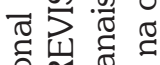

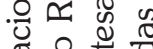

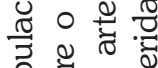

ठุ.

का के क्ष

㐘 000

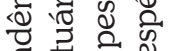

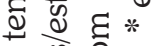

Q

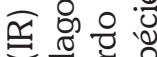

की

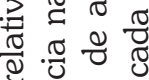

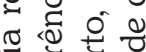

芯

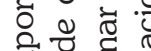

छ. 뜽

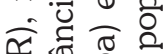

क

₹

हิ

$\frac{1}{2}$
$\frac{0}{8}$
$\frac{0}{0}$

\begin{tabular}{|c|c|c|c|c|c|c|c|c|c|c|c|c|c|c|c|c|c|c|c|c|c|c|}
\hline ฐ & $\Rightarrow$ & & & & & & & & & & & & & & & 0 & & 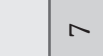 & $\infty$ & 0 & + & $\sigma$ \\
\hline$\leftarrow$ & $\vec{b}$ & & & & & & & & & & & & & & & 0 & & જे & 0 & $\stackrel{8}{\circ}$ & $\stackrel{L}{\sim}$ & 0 \\
\hline$"$ & tó & & & & & & & & & & & & & & & 0 & & $\vec{\sigma}$ & 0 & 0 & 0 & $\underset{-}{\stackrel{-}{2}}$ \\
\hline$\rightarrow$ & $\stackrel{\infty}{\sim}$ & & & & & & & & & & & & & & & 0 & & 0 & 8 & 0 & $\stackrel{2}{2}$ & 0 \\
\hline$\stackrel{\widehat{\varrho}}{\cong}$ & $F$ & & & & & & & & & & & & & & & $\hat{\circ}$ & & $\stackrel{m}{-}$ & $\stackrel{\sim}{ \pm}$ & $\hat{\sigma}$ & $\begin{array}{l}\dot{0} \\
\stackrel{v}{*}\end{array}$ & 0 \\
\hline$\approx$ & $\ddot{m}$ & & & & & & & & & & & & & & & $\infty$ & & 누 & $\Rightarrow$ & L & $\sim$ & 0 \\
\hline ఏิ & $\Rightarrow$ & 0 & $a$ & $\wedge$ & 0 & 0 & 0 & $\sigma$ & 10 & $\llcorner 0$ & $\infty$ & \llcorner & n & $\sigma$ & เ & $\wedge$ & $\wedge$ & \llcorner & & & & \\
\hline$\leftarrow$ & $\stackrel{-}{\circ}$ & 0 & 0 & 0 & 0 & 0 & 0 & 0 & 0 & 0 & $\begin{array}{l}\stackrel{L}{\infty} \\
\infty \\
\infty\end{array}$ & 0 & 0 & 0 & 0 & 0 & 00 & 0 & & & & \\
\hline " & 0 & 0 & 0 & 0 & 0 & $\begin{array}{l}m \\
m^{2}\end{array}$ & 0 & 0 & 0 & 0 & 0 & 0 & 0 & 0 & 0 & 0 & 0 & 0 & & & & \\
\hline$\rightarrow$ & 0 & $\underset{-}{\stackrel{0}{2}}$ & $\underset{-}{\stackrel{-}{2}}$ & $\stackrel{8}{\circ}$ & $\underset{-}{8}$ & हैं & $\underset{-}{8}$ & $\underset{-}{8}$ & $\underset{-1}{8}$ & $\stackrel{8}{\circ}$ & 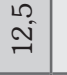 & 움 & 음 & 움 & 움 & \& & $\underset{-1}{8}$ & \& & & & & \\
\hline $\begin{array}{l}\stackrel{\widehat{o}}{0} \\
\cong\end{array}$ & $\begin{array}{l}L \\
\dot{m} \\
\dot{m}\end{array}$ & $\begin{array}{l}\vec{N} \\
\vec{N}\end{array}$ & $\stackrel{L}{\sigma}$ & $\begin{array}{l}\infty \\
\infty^{\infty}\end{array}$ & $\vec{\therefore}$ & $\begin{array}{l}\infty \\
+ \\
+\end{array}$ & $\begin{array}{l}\infty \\
+ \\
+\end{array}$ & $\stackrel{\vec{N}}{\mathrm{~s}}$ & $\stackrel{\vec{N}}{\mathrm{a}}$ & $\stackrel{\vec{v}}{\mathrm{i}}$ & $\stackrel{\sim}{\sim}$ & $\stackrel{\sim}{\sim}$ & 0 & 0 & 0 & 0 & 0 & 0 & & & & \\
\hline$\simeq$ & ลे & $\stackrel{\infty}{\sim}$ & $\infty$ & $\sim$ & 0 & $\sigma$ & $\sigma$ & $\sim$ & $\sim$ & $\sim$ & $\rightarrow$ & $\rightarrow$ & 0 & 0 & 0 & 0 & 0 & 0 & & & & \\
\hline 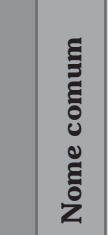 & 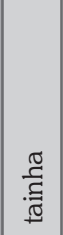 & 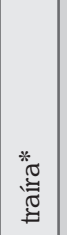 & 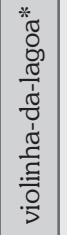 & 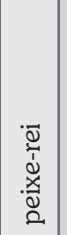 & 范 & 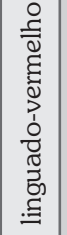 & 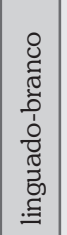 & 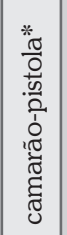 & 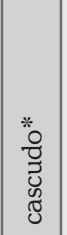 & 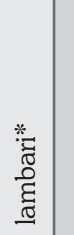 & छ亏 & 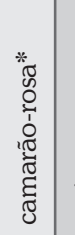 & : & : & 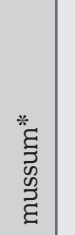 & $\begin{array}{l}\frac{0}{0} \\
\frac{0}{0} \\
00 \\
0\end{array}$ & \begin{tabular}{l|l} 
& 0 \\
& 0 \\
0
\end{tabular} & 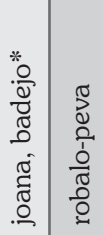 & 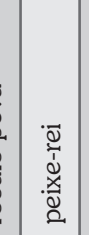 & 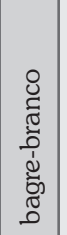 & 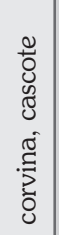 & 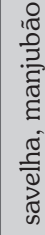 \\
\hline 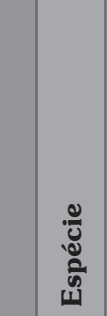 & 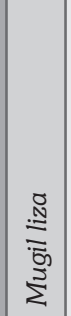 & 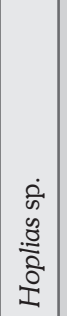 & 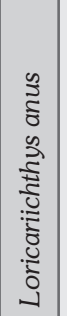 & 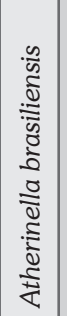 & 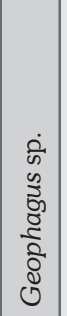 & 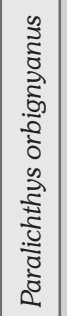 & 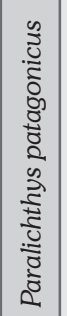 & 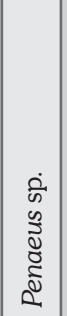 & 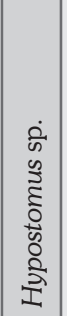 & 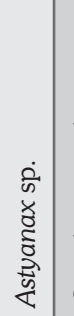 & 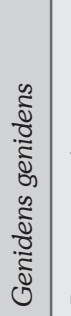 & 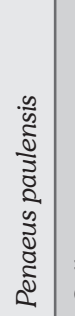 & 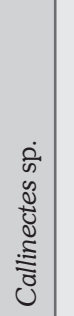 & 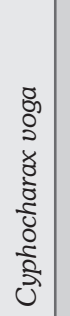 & 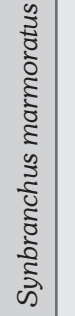 & 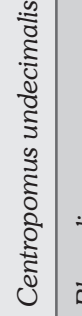 & 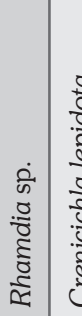 & 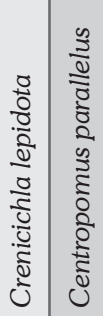 & 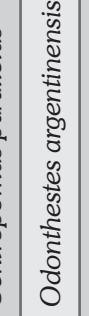 & 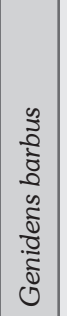 & 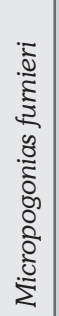 & 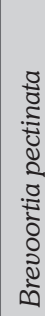 \\
\hline
\end{tabular}




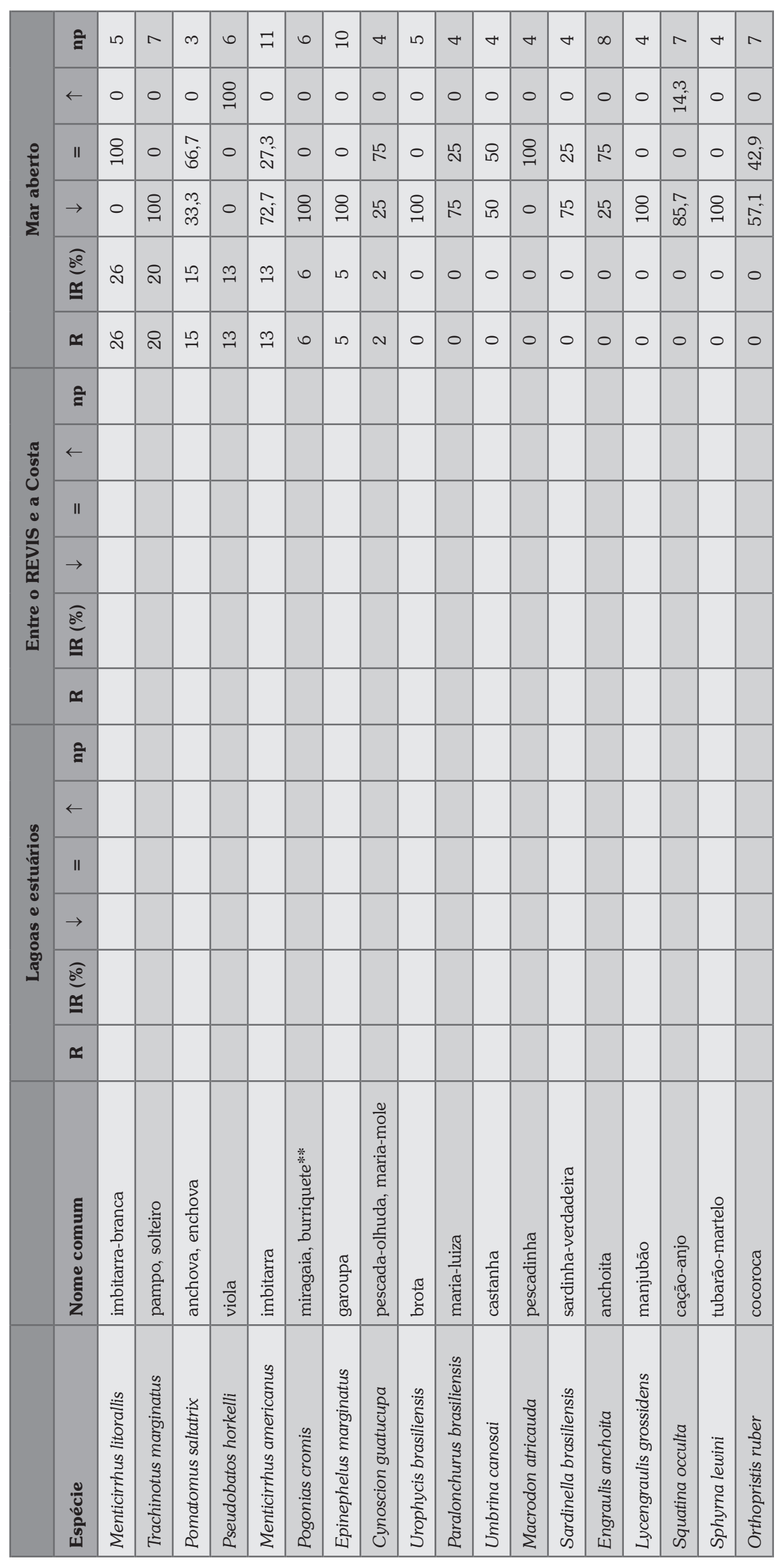




\section{Ameaças à ictiofauna, de acordo com pescadores artesanais da região do REVIS Ilha dos Lobos}

As ameaças identificadas estão descritas na Tabela 3 , de acordo com as regióes de predominância de ocorrência das espécies consideradas importantes.
Conforme indicado na Tabela 3, as ameaças às espécies avaliadas na oficina estão estreitamente correlacionadas às ameaças aos ambientes em que estas ocorrem, além das ameaças relacionadas à pesca desordenada ou excessiva. A redução dessas ameaças depende, por isso, tanto da adequação do ordenamento pesqueiro quanto do ordenamento territorial, sendo fundamental, para isso, o amplo envolvimento social e institucional.

Tabela 3 - Ameaças a espécies da ictiofauna da região do REVIS llha do Lobos, por região predominante de ocorrência, de acordo com pescadores artesanais.

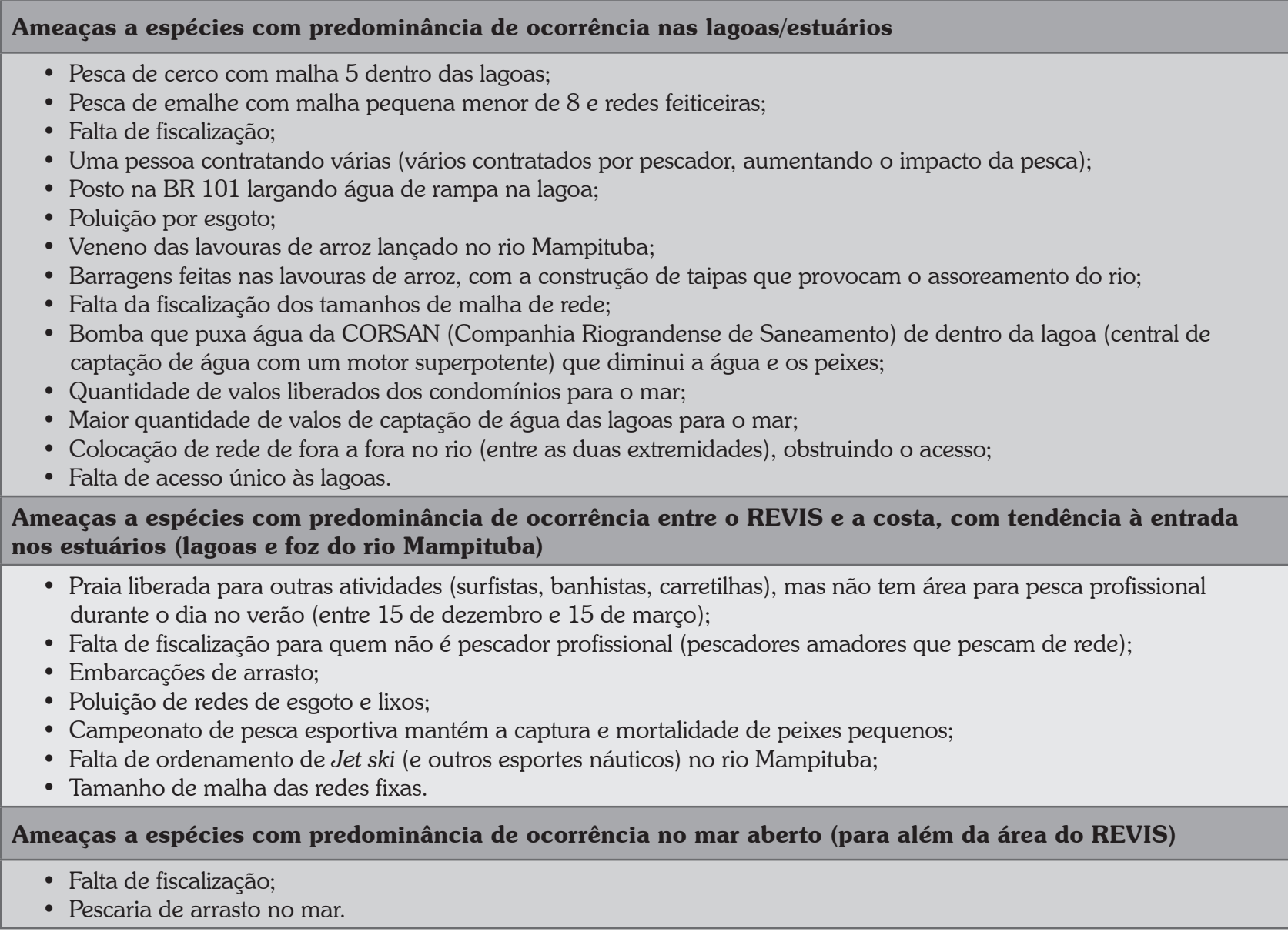

\section{Conclusão}

Agregar o conhecimento ecológico local na gestão das áreas protegidas, além de reconhecer e valorizar os diferentes saberes em relação à biodiversidade local, é uma estratégia para integrar a gestão das UCs ao seu território.

Nesse sentido, a metodologia pode ser reaplicada periodicamente, no âmbito da gestão da UC, para avaliar a tendência populacional de determinadas espécies ao longo do tempo, tanto pela sua importância comercial quanto biológica, sobretudo com complementações de ferramentas que consigam adequar o tempo geracional das várias espécies envolvidas, que possui intrínseca relação com a capacidade de reposição populacional. Variações na avaliação da tendência populacional dessas espécies podem servir como indicadores, inclusive, de efetividade de medidas de gestão. 
No contexto de gestão do REVIS Ilha dos Lobos, a metodologia permitiu balizar os levantamentos prévios a partir de dados secundários da bibliografia, bem como indicar novos registros a partir da experiência dos pescadores artesanais da região. Além disso, a partir dos seus saberes, os pescadores têm suas hipóteses para as causas de declínio ou aumento populacional de uma determinada espécie. Considerar esse conhecimento na gestão da UC, agregando conhecimentos técnico-científicos, significa fortalecer os espaços de participação social e focar em estratégias de gestão e políticas públicas que sejam mais adequadas a cada território, integrando esforços para o diagnóstico e o enfrentamento das ameaças à conservação.

Além disso, a metodologia - baseada na oralidade ao invés da exposição, na utilização de imagens das espécies e na construção conjunta do mapa - facilitou a interlocução com os pescadores, em especial os com dificuldade de leitura escrita. Assim, recomenda-se que sejam utilizados, em situações análogas, materiais visuais comunicativos para facilitar a compreensão entre todos os presentes.

Idealmente, o período de tempo da oficina deveria ser expandido, a fim de viabilizar a identificação online e em conjunto da nomenclatura científica daquelas espécies não identificadas previamente para checklist, possibilitar a utilização de "linhas do tempo" e "histórias orais" para a avaliação de tendência populacional de cada espécie em maiores períodos de tempo e, especialmente, possibilitar maior debate sobre a tendência populacional $e$ as ameaças às espécies envolvidas, sobretudo quando possuem diferentes tempos geracionais.

A possibilidade de aplicação da metodologia aqui descrita como contribuição ao processo de avaliação do estado de conservação das espécies de uso se manifesta tanto na identificação da ocorrência das espécies quanto na análise da tendência populacional e das ameaças. Os manuais da IUCN admitem a incorporação de dados de várias tipologias nas avaliações de estado de conservação, inclusive qualitativos, que inferem certas tendências a partir de outras percepções de diminuição, aumento ou estabilidade do tamanho populacional (IUCN 2019).

É importante notar que as análises por meio do CEL, além de estruturar os diferentes saberes para integrar os processos de avaliação, podem indicar contrapontos em relação à literatura tradicional. Como exemplos, a viola e o bagre-branco, categorizados oficialmente como criticamente em perigo (CR) e em perigo (EN), respectivamente (ICMBio 2018), ou ainda a tainha (categorizada como sobre-explotada) (Sant'ana \& Kinas 2015), foram caracterizados pelos pescadores, neste trabalho, com um aumento na sua tendência populacional, ao menos em algum ambiente. É possível que isso ocorra, em parte, em função da subjetividade do período de tempo para o qual se propôs a avaliação da tendência populacional, ou mesmo em função da percepção mais voltada à última safra, que tende a estar mais presente na memória imediata dos pescadores. No caso da tainha, por exemplo, a safra de 2018 tem sido reportada como atípica, ou mesmo como supersafra (Steenbock 2019). É importante considerar, todavia, que na avaliação do estado e conservação das espécies é comum a análise de trabalhos científicos que apontam, para a mesma espécie, tendências populacionais distintas. Assim, é justamente a análise de diferentes abordagens e trabalhos, de forma qualificada, que permite a categorização do estado de conservação das espécies. E, nesse conjunto de abordagens, é importante a agregação de sistematizações do CEL e o aprimoramento de sua aplicação contínua. Dessa forma, as informações obtidas e sistematizadas pelo conjunto de pescadores podem ser consideradas no conjunto de informações disponíveis para avaliar o risco de extinção dessas espécies, tornando o processo ainda mais completo $e$ inclusivo. E, em sendo aplicada em diferentes UCs, pode viabilizar uma contribuição importante do conhecimento ecológico local nesse sentido.

Os resultados indicam, por fim, que independentemente da categoria de conservação da UC em questão, fazer a gestão considerando o envolvimento direto, qualificado e proativo dos atores comunitários nas decisões de gestão tende a contribuir para o alcance de suas metas de conservação.

\section{Referências Bibliográficas}

Begossi A, Salivonchyk S, Hallwass G, Hanazaki N, Lopes PFM \& Silvano RAM. Threatened fish and fishers along the Brazilian Atlantic Forest Coast. AMBIO, v46, p. 907-914, 2017.

Brasil. 1998. Decreto n 2.519, de 16 de março de 1998. Diário Oficial da União. http:/www.planalto.gov.br/ ccivil_03/decreto/D2519.htm\#: :text=Promulga\%20 
a\%20Conven \%C3\%A7\%C3\%A3o\%20sobre\%20 Diversidade,que\%20lhe\%20confere\%20o\%20art. Acesso em: 01/09/2020.

Brasil. 2000. Lei $\mathrm{n}^{\circ} 9.985$ de 18 de julho de 2000. Diário Oficial da União. http://www.planalto.gov.br/ ccivil_03/leis/19985.htm. Acesso em: 01/09/2020.

Brasil. 2002. Decreto $n^{\circ} 4340$, de 22 de agosto de 2002. Diário Oficial da União. http://www. planalto.gov.br/ccivil_03/decreto/2002/D4340. htm\#: : text $=$ Regulamenta\%20artigos\%20da\%20 Lei $\% 20$ n,que\%20lhe\%20conferem \%20o\%20art. Acesso em: 01/09/2020.

Brasil. 2014a. Portaria MMA n ${ }^{\circ} 43$ de 31 de janeiro de 2014. Diário Oficial da União. https://www.icmbio.gov.br/ portal/images/stories/docs-plano-de-acao-ARQUIVO/00saiba-mais/03_-_PORTARIA_MMA_N\%C2\%BA_43 DE_31_DE_JAN__DE_2014.pdf. Acesso em: 01/09/2020.

Brasil. 2014b. Portaria MMA no 349 de 26 de setembro de 2014. Diário Oficial da União. http://www.lex.com. br/legis_26012431_PORTARIA_N_349_DE_26_DE_ SETEMBRO_DE_2014.aspx. Acesso em: 01/09/2020.

Brose M. (org). 2001. Metodologia participativa: uma introdução a 29 instrumentos. Tomo Editorial. 312p.

Diegues AC. (org.). 2000. Etnoconservação: novos rumos para a proteção da natureza nos trópicos. 2 ed. Hucitec/Annablume. 290p.

Geilfus F. 2002. 80 herramientas para el desarrollo participativo: diagnóstico, planificación, monitoreo, evaluación. IICA. 217p.

Hanazaki N, Berkes F, Seixas CS \& Peroni N. Livelihood Diversity, Food Security and Resilience among the Caiçara of Coastal Brazil. Humam Ecology, 41: 153-164, 2013

ICMBio (Instituto Chico Mendes de Conservação da Biodiversidade). 2018. Livro Vermelho da Fauna Brasileira Ameaçada de Extinção: Volume I. 1 ed. ICMBio. 492p.
ICMBio (Instituto Chico Mendes de Conservação da Biodiversidade). 2013. Instrução Normativa $n^{\circ} 34$ de 17 de outubro de 2013. Diário Oficial da União. https:// www.icmbio.gov.br/ran/images/stories/legislacao/in icmbio_34_2013_diretrizes_avalia\%C3\%A7\%C3\%A3o_ estado_conserva\%C3\%A7\% C3\%A3o_fauna_e_sistema_ esp\%C3\%A9cies.pdf. Acesso em: 01/09/2020.

IUCN. 2019. Guidelines for Using the IUCN Red List Categories and Criteria. Version 14. IUCN. 113p.

Kellermann A. 2018. Refúgio de Vida Silvestre da Ilha dos Lobos: formação e caracterização do seu conselho gestor. Dissertação (Mestrado Profissional em Ambiente e Sustentabilidade). Universidade Estadual do Rio Grande do Sul. 92p.

Minayo MC de S (org). 1994. Pesquisa social: teoria, método e criatividade. Vozes. 80p.

Sant'Ana R \& Kinas PG. 2015. Avaliação do estoque de tainha (Mugil liza): ampliação dos modelos bayesianos de dinâmica de biomassa para múltiplas séries de CPUE, com adição de temperatura superficial do mar e capturabilidade autocorrelacionada. Relatório II Avaliação de estoque - Tainha - OCEANA. 31p.

Souza ECF, Brant A, Rangel CA, Barbosa LE, Carvalho CEG, Jorge RSP \& Subirá RJ. Avaliação do risco de extinção da fauna brasileira: ponto de partida para a conservação da biodiversidade. Diversidade e Gestão 2(2): 62-75, 2018.

Steenbock W \& Medeiros RP. 2017. Manual de avaliação e monitoramento socioeconômico para a gestão de recifes de coral. Instituto Chico Mendes de Conservação da Biodiversidade. 274p. Traduzido de Bunce L, Townsley P, Pomeroy R \& Pollnac R. 2000. Global Coral Reef Monitoring Network, Australian Inst. Marine Science. 251p.

Steenbock W. Subsídios para o ordenamento da pesca da tainha (Mugil liza, Mugilidae) uma análise histórica recente de aspectos relacionados à política de cotas. Biodiversidade e conservação marinha, 8: 1-30, 2019.

Biodiversidade Brasileira - BioBrasil.

Fluxo Contínuo

n. 3,2020

http://www.icmbio.gov.br/revistaeletronica/index.php/BioBR

Biodiversidade Brasileira é uma publicação eletrônica científica do Instituto Chico Mendes de

Conservação da Biodiversidade (ICMBio) que tem como objetivo fomentar a discussão e a disseminação de experiências em conservação e manejo, com foco em unidades de conservação $e$ espécies ameaçadas.

ISSN: 2236-2886 